\title{
Hierarchical Clustering on RNA Dependent RNA Polymerase using Machine Learning.
}

\author{
GUDIPATI.PAVAN KUMAR* \\ * Independent Researcher, Hyderabad, India.
}

\begin{abstract}
RNA Dependent RNA Polymerase (RdRP) catalyzes the replication of RNA from an RNA template and is mostly found in Viruses. We have collected over 161 viral RdRP FASTA Sequences from the NCBI protein database using python script. Each of these sequences was transformed with TfidfVectorizer using sklearn module, with the "one Letter" word, because each Letter belongs to one Amino acid. These transformed data were sent to Hierarchical clustering using scipy library and visualized data using Dendrogram. These Machine Learning technique is able to classify or segment similar RdRp into one cluster. Each of these clusters was tested for their multiple sequence alignment with COBALT of NCBI. We observed that these clusters predicted similar RdRP among various viruses. These techniques can be further improved to segment or classify various proteins. These Machine Learning or Artificial Intelligence techniques need more improvement in their algorithms to solve genomics and proteomics.
\end{abstract}

Keywords: RNA Dependent RNA Polymerase, RdRP, Machine Learning, Hierarchical Clustering, sklearn, scipy, TfidfVectorizer, Dendrogram, Biopython

Repository: Programs and Datasets : https://github.com/DSPavan/RdRP

\section{INTRODUCTION}

RNA-dependent RNA polymerase (RdRp) is an enzyme that catalyzes the replication of RNA from an RNA template. RNA dependent RNA polymerase (RdRp) of SARS COV-2 is known to mediate replication of the viral genome and its propagation inside host cells [1]. Several RNA containing Viruses have this enzyme. Hierarchical clustering, also known as hierarchical cluster analysis, is an algorithm that groups similar objects into groups called clusters. The endpoint is a set of clusters, where each cluster is distinct from each other cluster, and the objects within each cluster are broadly similar to each other. [2,3]. NCBI Protein database is a collection of sequences from several sources, including translations from annotated coding regions in GenBank, RefSeq and TPA, as well as records from SwissProt, PIR, PRF, and PDB. [4]. Python is a programing language widely used in Machine Learning, and they have sklearn [5] and scipy [6] modules. Main aim of this experiment is to test, can we cluster similar RdRP with help of Machine Learning / Artificial Intelligence techniques?

\section{MATERIAL \& METHODS}

A) Collection of RdRP: Primarily this data collected from NCBI website, under Protein Database. These data can be collected manually from this database, using search option. We can also Biopython modules to get all these data from programmatically. I have extracted RdRP data using Biopython [7] with Entrez [8] module, specifically Amino acid sequences length between 600 to 3500 and excluded partial, probable, putative types from the list, so that we will get clean records. I have used kaggle notebook [9] with Python, which have preinstalled biopython libraries. I have collected 490+ RdRP from various organisms, mostly Viruses. After extraction of data, manually curation is done, to remove duplicates or identical proteins. After final curation we have dataset of 161 RdRP proteins.

Biopython CODE:

from Bio import Entrez

Entrez.email = "example@example.org" 
searchResultHandle = Entrez.esearch $(\mathrm{db}=$ "protein", term= "RNA-dependent RNA polymerase[Title] AND RNAdependent RNA polymerase[Protein Name] AND 600:3500[Sequence Length] NOT ( partial OR probable OR TPA_asm O R pdb OR like OR eukaryotic-type OR putative OR TPA OR mitoviral OR like)", retmax=500)

searchResult $=$ Entrez.read $($ searchResultHandle $)$

ids = searchResult["IdList"]

\#print(ids)

handle $=$ Entrez.efetch $(\mathrm{db}=$ "protein", $\mathrm{id}=\mathrm{ids}$, retmax=500, rettype="fasta", retmode="text")

record $=$ handle.read ()

\# The output file will be saved under Dataset/output section

out_handle = open('RdRP3.fasta', 'w')

out_handle.write(record.rstrip('ไn'))

\#print(record)

B) TfidfVectorizer: Each Fasta sequences of RdRP was transformed with TfidfVectorizer method with in sklearn module. TfidfVectorizer, Convert a collection of raw documents to a matrix of TF-IDF features. In this experiment, we specifically tried with single letter word, so that, each amino acid in Fasta sequence can be transformed properly with above matrix.

\section{\#code}

from sklearn.feature_extraction.text import TfidfVectorizer

vectorizer $=$ TfidfVectorizer(stop_words $=\{$ 'english' $\}$, analyzer='char', ngram_range $\left.=(1,1), \min \_\mathrm{df}=1, \mathrm{max} \_\mathrm{df}=1.0\right)$

C) Using Kmeans: Number of optimum clusters were identified with Elbow technique and silhouette coefficients. Optimum Cluster to get all 161 Fasta sequences was 10 to 20 clusters.

D) Hierarchical Cluster: After TfidfVectorizer, this data clustered with Linkage type "ward". Then it is converted into Dendogram tree. After verifying dendrogram results, we can observe 8 major clusters, with color_threshold=0.14, each of these can be given unqiue color, so that, we can analyze each cluster for further analysis. You can vary color threshold, so that, you can verify different cluster sizes. I have kept this value (0.14), so that we can optimum size of clusters for analysis. With Cluster size-15, all 161 sequences clearly assigned to specific cluster.

\section{\#code}

Zx = linkage(AAVector.toarray(), 'ward')

from scipy.cluster.hierarchy import dendrogram, fcluster, leaves_list, set_link_color_palette set_link_color_palette([ "green","red","blue",,orange","black","brown","Teal","Magenta"])

dend $=$ dendrogram $(Z x$, color_threshold=0.14,leaf_font_size=12,labels=fheaderList, orientation='right', distance_sort='desce nding',truncate_mode='level',no_plot=True)

E) Extraction of Each Cluster for Further Analysis: We will get complete Denogram from above code, We need to extract, each color Cluster, using fcluster module, and we can extract list of fasta sequences of RdRP, which are grouped in same cluster.

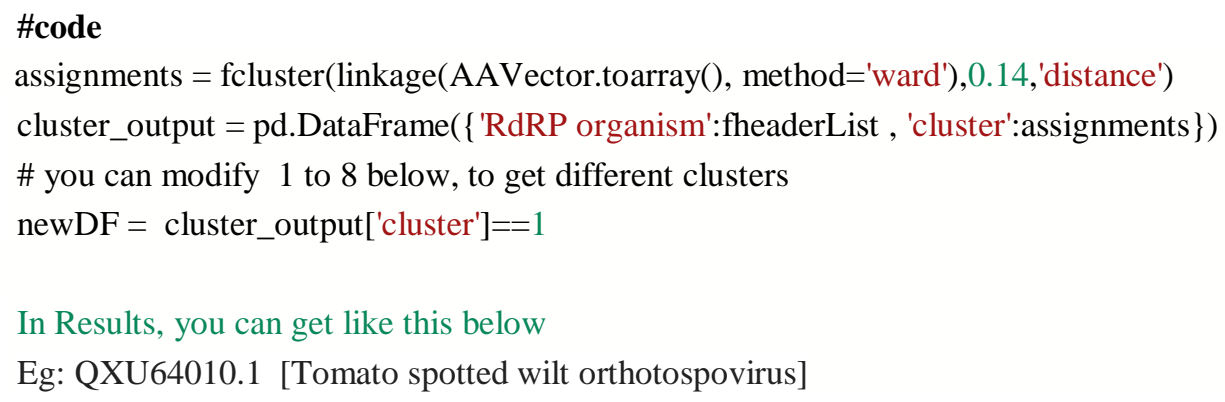


F) Constraint-based Multiple Alignment Tool of NCBI: Each of these clusters, which we extracted from above, was given as input ( Accession Number is sufficient) into COBALT (Constraint-based Multiple Alignment Tool of NCBI) [10, 11]. After alignment, set Conservation Setting as "Identity". We collected, near to cluster branch as in dendrogram tree results, and send again for alignment, and we got below results.

\section{RESULTS AND DISCUSSION}

Each of these clusters sequences are aligned with COBALT- (Constraint-based Multiple Alignment Tool) with Conservation Setting as Identity.

\section{A. Green Cluster}

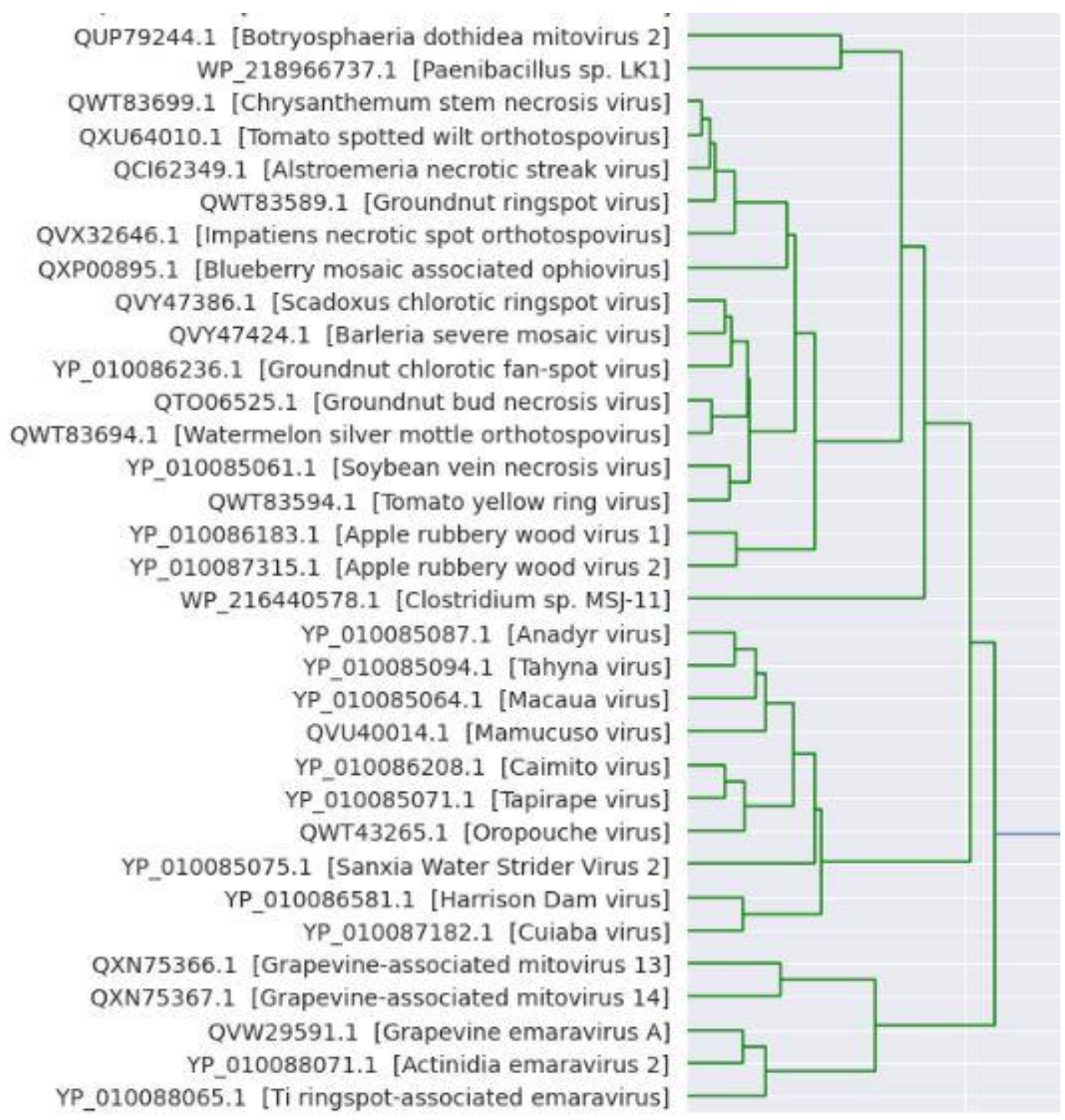

Fig. 1: Green Cluster. 
Table 1: Green Cluster (Cluster No: 1) : Highly matching cluster

\begin{tabular}{|l|l|l|}
\hline Set & Accession No. & Virus \\
\hline A & QXU64010.1 & Tomato Spotted Wilt Orthotospovirus \\
\hline A & QWT83699.1 & Chrysanthemum Stem Necrosis Virus \\
\hline A & QWT83589.1 & Groundnut Ringspot Virus \\
\hline A & QCI62349.1 & Alstroemeria Necrotic Streak Virus \\
\hline & & \\
\hline B & QWT83694.1 & Watermelon Silver Mottle Orthotospovirus \\
\hline B & QTO06525.1 & Groundnut Bud Necrosis Virus \\
\hline & & \\
\hline C & QVW29591.1 & Grapevine Emaravirus A \\
\hline C & YP_010088071.1 & Actinidia Emaravirus 2 \\
\hline
\end{tabular}

B. Red Cluster
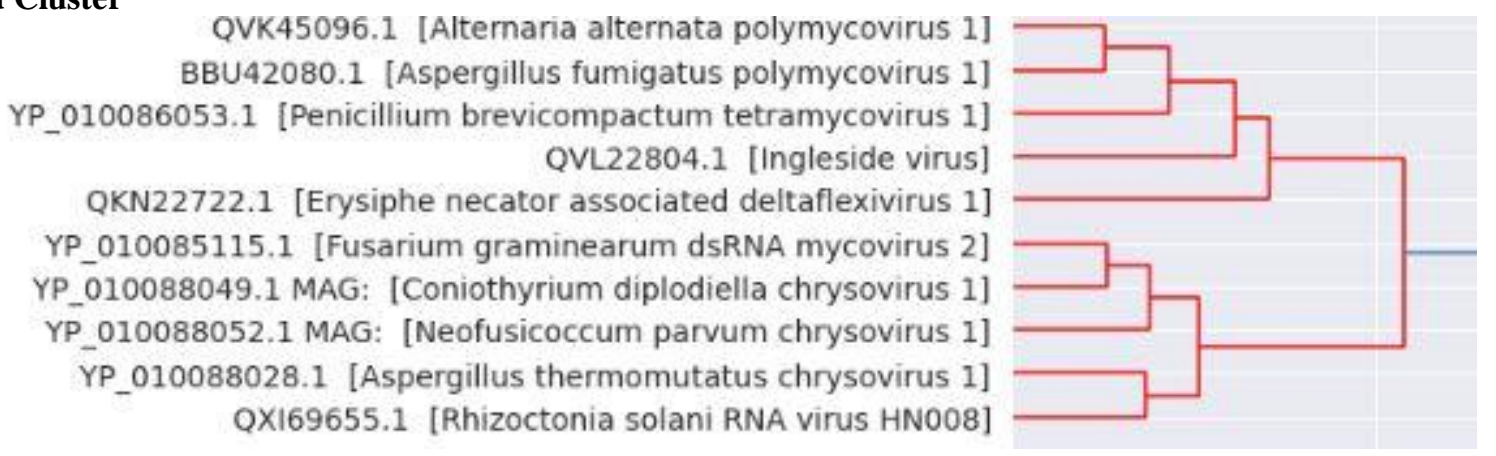

Fig. 2: Red Cluster.

Table 2: Red Cluster (Cluster No: 2): Highly matching cluster

\begin{tabular}{|l|l|l|}
\hline Set & Red & Virus \\
\hline A & BBU42080.1 & Aspergillus fumigatus polymycovirus 1 \\
\hline A & QVK45096.1 & Alternaria alternata polymycovirus 1 \\
\hline & & \\
\hline B & YP_010085115.1 & Fusarium graminearum dsRNA mycovirus 2 \\
\hline B & YP_010088049.1 & Coniothyrium diplodiella chrysovirus 1 \\
\hline
\end{tabular}

C. Blue Cluster:

Table 3: Blue Cluster (Cluster No: 3) Highly matching cluster

\begin{tabular}{|l|l|l|}
\hline Set & Accession No. & Virus \\
\hline A & QWB49515.1 & Carnation Italian Ringspot Virus \\
\hline A & QVX32680.1 & Cymbidium Ringspot Virus \\
\hline A & QVX32675.1 & Tomato Bushy Stunt Virus \\
\hline & & \\
\hline B & QWC36209.1 & Pelargonium Necrotic Spot Virus \\
\hline B & QWC36204.1 & Pelargonium Leaf Curl Virus \\
\hline
\end{tabular}


bioRxiv preprint doi: https://doi.org/10.1101/2021.08.23.457366; this version posted August 23, 2021. The copyright holder for this preprint (which was not certified by peer review) is the author/funder, who has granted bioRxiv a license to display the preprint in perpetuity. It is made available under aCC-BY-NC-ND 4.0 International license.

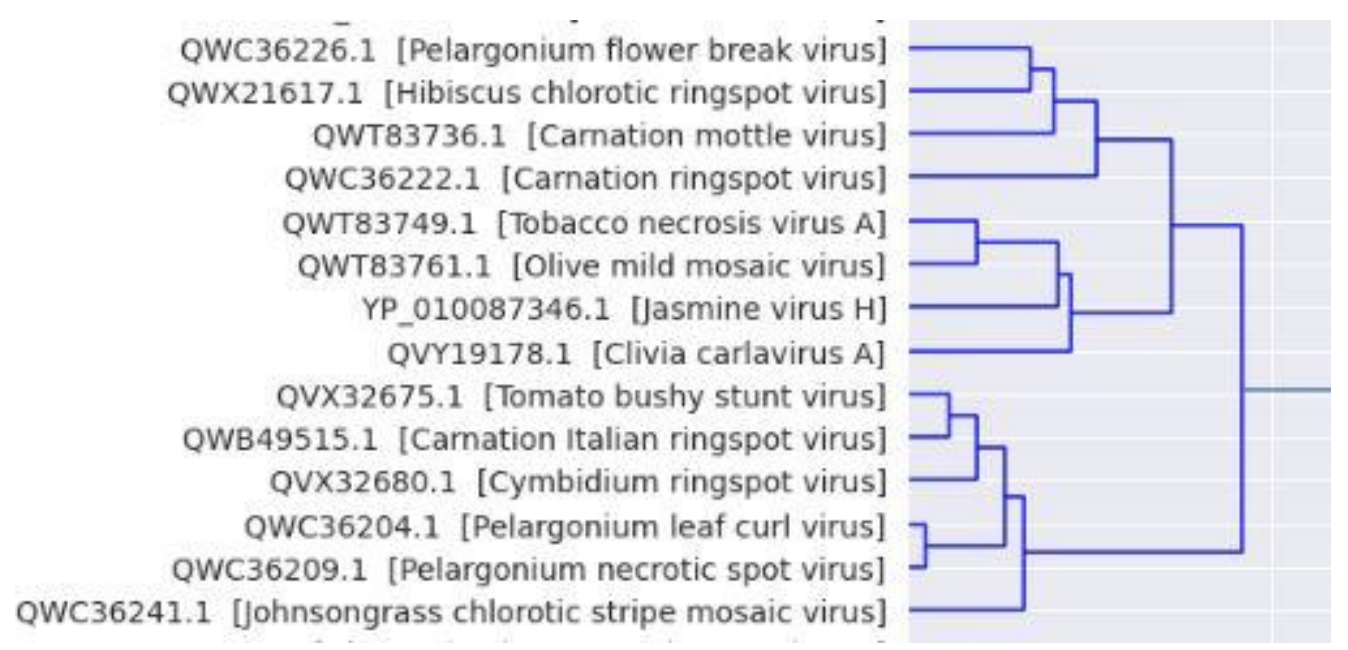

Fig. 3: Blue Cluster.

D. Orange Cluster:
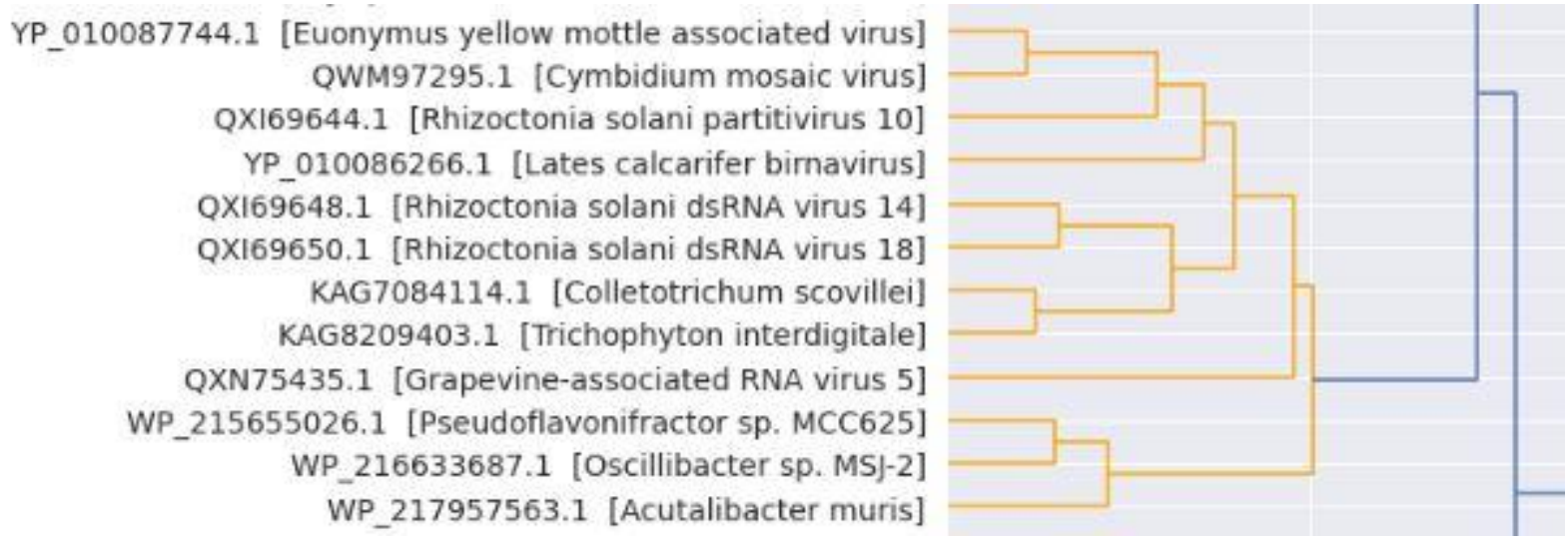

Fig. 4: Orange Cluster.

Table 4: Orange Cluster (Cluster No: 4): Highly matching cluster

\begin{tabular}{|l|l|l|}
\hline Set & Accession No. & Virus \\
\hline A & QWM97295.1 & Cymbidium mosaic virus \\
\hline A & YP_010087744.1 & Euonymus yellow mottle associated virus \\
\hline B & QXI69650.1 & Rhizoctonia solani dsRNA virus 18 \\
\hline B & QXI69648.1 & Rhizoctonia solani dsRNA virus 14 \\
\hline & & \\
\hline C & WP_216633687.1 & Oscillibacter sp. MSJ-2 \\
\hline C & WP_215655026.1 & Pseudoflavonifractor sp. MCC625 \\
\hline & & \\
\hline D & KAG8209403.1 & Trichophyton interdigitale \\
\hline D & KAG7084114.1 & Colletotrichum scovillei \\
\hline
\end{tabular}




\section{E. Black Cluster:}

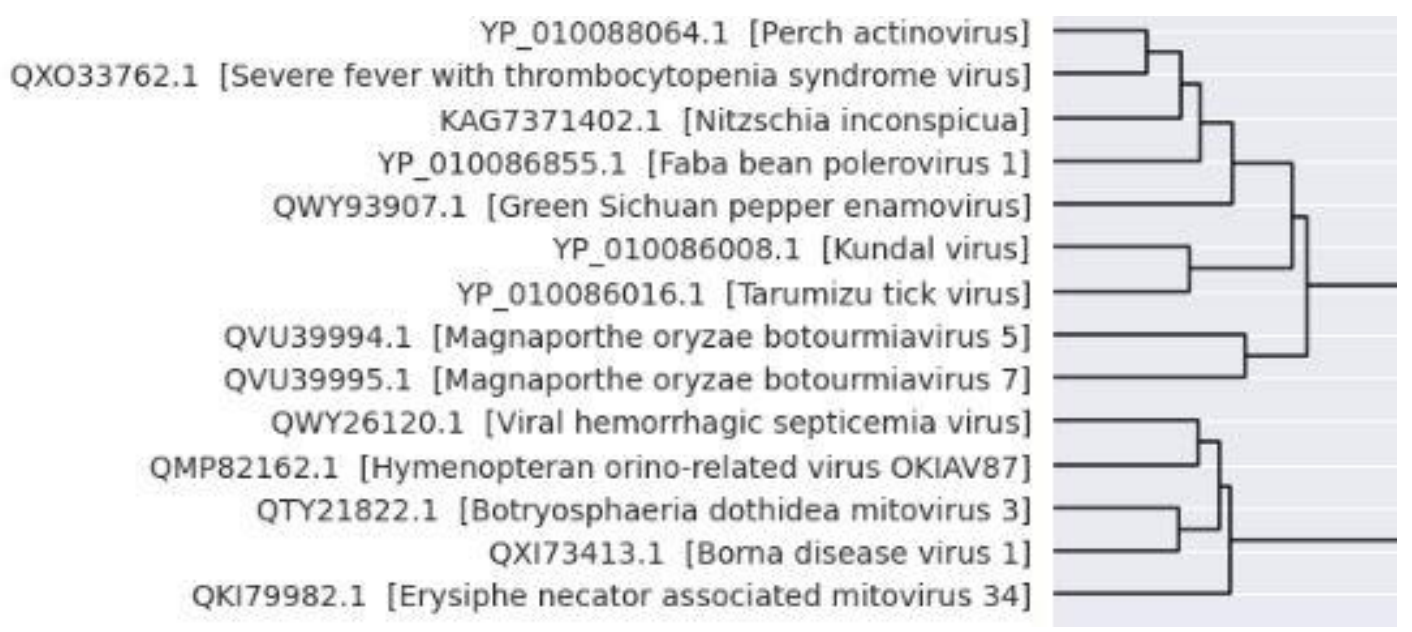

Fig. 5: Black Cluster.

Table 5: Black Cluster (Cluster No: 5) : Highly matching cluster

\begin{tabular}{|l|l|l|}
\hline Set & Accession No. & Virus \\
\hline A & YP_010086016.1 & Tarumizu Tick Virus \\
\hline A & YP_010086008.1 & Kundal Virus \\
\hline & & \\
\hline
\end{tabular}

Note: There is no good match with other sequences, and huge difference is observed, probably because of long distance between them.

\section{F. Brown Cluster:}

Table 6: Brown Cluster (Cluster No: 6) : Highly matching cluster

\begin{tabular}{|l|l|l|}
\hline Set & Accession No. & Virus \\
\hline A & QXJ13539.1 & Mokola Lyssavirus \\
\hline A & QIK01206.1 & Rabies Lyssavirus \\
\hline A & YP_010086774.1 & Taiwan Bat Lyssavirus \\
\hline
\end{tabular}




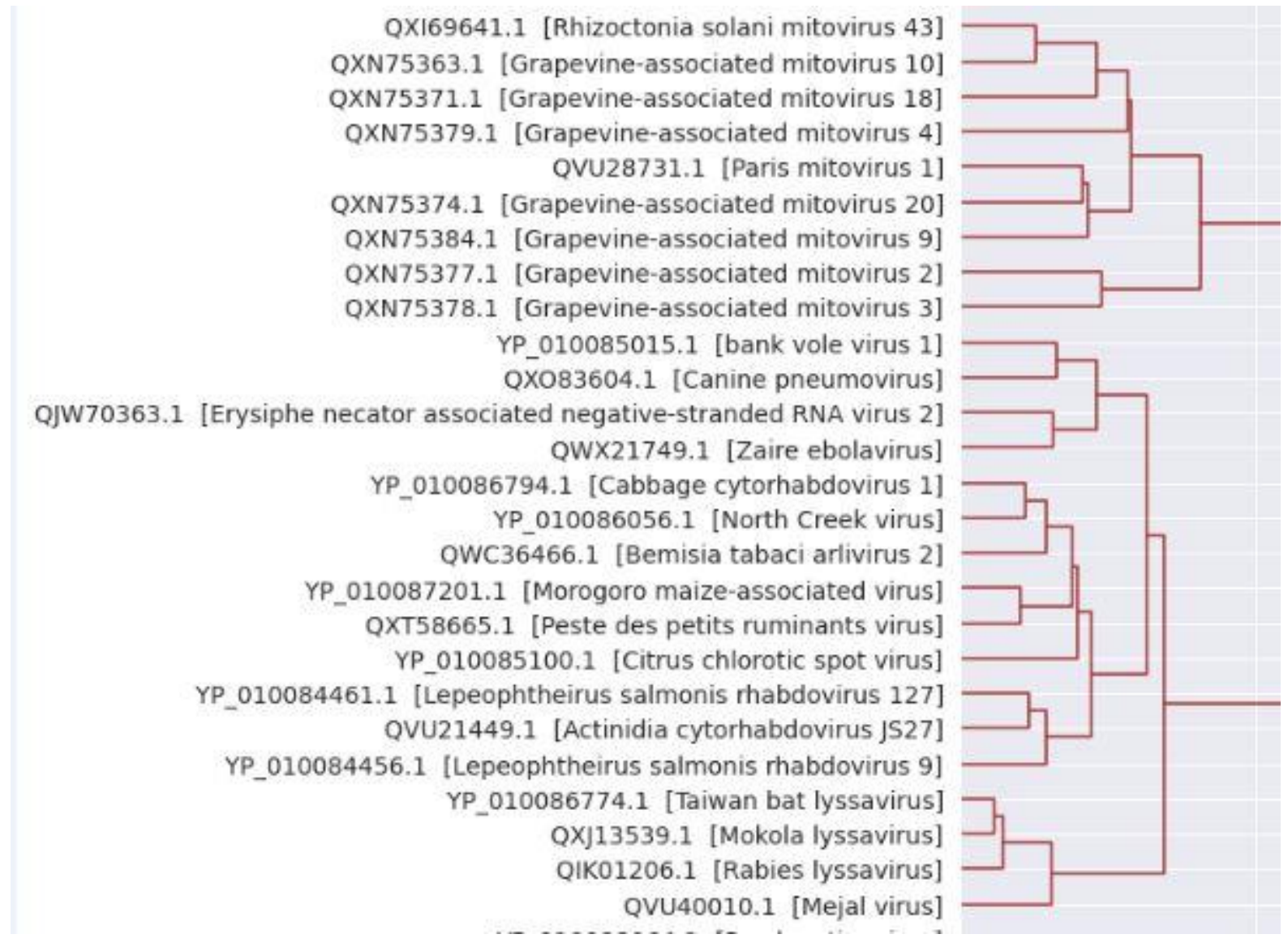

Fig. 6: Brown Cluster.

\section{G. Teal Cluster:}

QWX94191.1 [Aedes aegypti totivirus 2]

QXN75344.1 [Grapevine-associated fusarivirus 1]

QWT83704.1 [Sunn-hemp mosaic virus] QWT83775.1 [Odontoglossum ringspot virus] QWT83771.1 [Youcai mosaic virus]

QVX32625.1 [Tomato mottle mosaic virus] QWT83680.1 [Tobacco mosaic virus] QXT50807.1 [Tomato brown rugose fruit virus] YP_010088081.1 [Passion fruit green spot virus] QWT83571.1 [Cucumber green mottle mosaic virus] QWX21611.1 [Hibiscus latent Fort Pierce virus] QWC36232.1 [Cucumber mosaic virus] QWT83606.1 [Brome mosaic virus] QWT83732.1 [Tomato aspermy virus] QWC36237.1 [Tobacco streak virus]

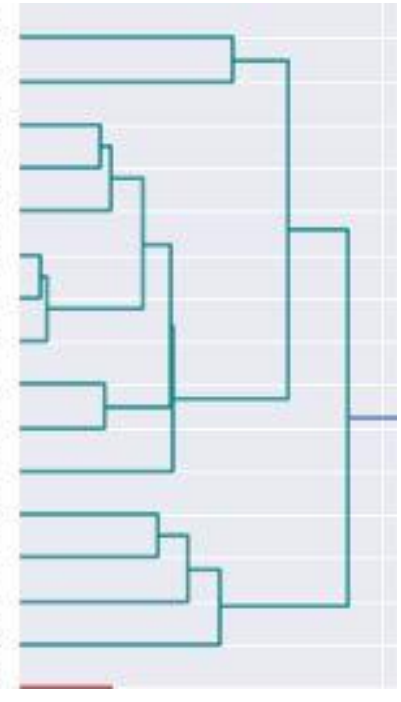

Fig. 7: Teal Cluster. 
Table 7: Teal Cluster (Cluster No: 7) : Highly matching cluster

\begin{tabular}{|l|l|l|}
\hline Set & Accession No. & Virus \\
\hline Set A & QWT83775.1 & Odontoglossum Ringspot Virus \\
\hline Set A & QWT83771.1 & Youcai Mosaic Virus \\
\hline Set A & QWT83704.1 & Sunn-Hemp Mosaic Virus \\
\hline & & \\
\hline Set B & QXT50807.1 & Tomato Brown Rugose Fruit Virus \\
\hline Set B & QWT83680.1 & Tobacco Mosaic Virus \\
\hline Set B & QVX32625.1 & Tomato Mottle Mosaic Virus \\
\hline
\end{tabular}

H. Magneta Cluster:

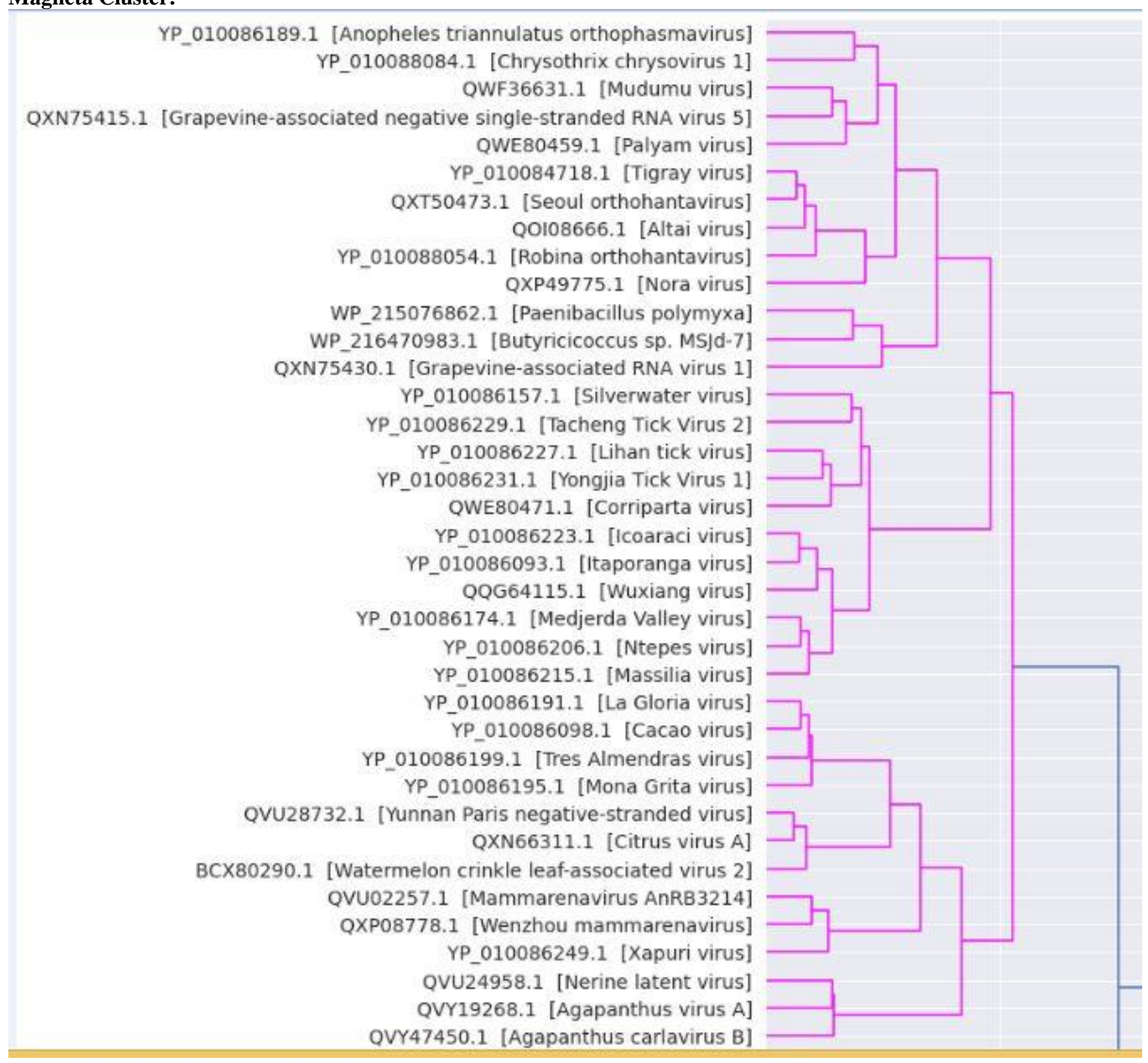

Fig. 8: Magneta Cluster. 
Table 8: Magneta Cluster (Cluster No: 8): Highly matching cluster

\begin{tabular}{|l|l|l|}
\hline Set & Accession No. & Virus \\
\hline Set A & YP_010086098.1 & Cacao Virus \\
\hline Set A & YP_010086191.1 & La Gloria Virus \\
\hline & & \\
\hline Set B & YP_010086093.1 & Itaporanga Virus \\
\hline Set B & YP_010086223.1 & Icoaraci Virus \\
\hline & & \\
\hline set C & QXP08778.1 & Wenzhou Mammarenavirus \\
\hline Set C & QVU02257.1 & Mammarenavirus Anrb3214 \\
\hline
\end{tabular}

\section{Constraint-based Multiple Alignment Tool output with Teal Cluster Set B as follows:}

\begin{tabular}{|c|c|c|c|}
\hline .1 & 1 & MAYTQTATTSALLDTVRGNNTLVNDLAKRRLYDTAVDEFNARDRRPKVNFSKV & 10 \\
\hline QWT 83680.1 & 1 & AYTQTATTSALLDTVRGNNSLVNDLAKRRLYDTAVDEFNARDRRPKVNFSKVISEEQTLIATRAYPEFQITFYNTQNAV & a \\
\hline ovx32625.1 & 1 & YTQTATSSALLDTVRGNNTLVNDLAKRRLYDTAVDEFNARDRRPKVNFSKVVSEEQTLIATRAYPEFQITFYNTQNAV & $\theta$ \\
\hline .1 & 1 & HSLAGGLRSLELEYLMMQIPYGSLTYDIGGNFASHLFKGRAYVHCCMPNLDVRDIMRHEGQKDSIELYLSRLERGNKVVP & \\
\hline 33680.1 & 81 & HSLAGGLRSLELEYLMMQVPYGSLTYDIGGNFASHLFKGRAYVHCCMPNLDVRDIMRHEGQKDSIELYLSRLERGNKQVP & 160 \\
\hline$\underline{32625.1}$ & 81 & HSLAGGLRSLELEYLMMQIPYGSLTYDIGGNFASHLFKGRAYVHCCMPNLDVRDIMRHEGQKDSVELYLARLERGNKFVP & 160 \\
\hline 97.1 & 161 & NFQKEAFDRYAETPDEVVCHSTFQTCTHQQVENTGRVYAIALHSIYDIPADEFGAALLRKNVHVCYAAFHFSENLLLEDS & \\
\hline QWT 83680.1 & 161 & AFQKEAFDRYAEVSNEVVCHNTFQTCEHQQMOHTGRVYAIALHSIYDIPADEFGAALLRKNVHTCYAAFHFSENLLLEDS & 40 \\
\hline 625.1 & 161 & NFQKEAFDRYAETPDEVVCHDTFQTCRHSQEMYTGRVYAIALHSIYDIPADEFGAALLRKNVHVCYAAFHFSENLLLEDS & 240 \\
\hline 0807.1 & 241 & DEINACFSRDGDKLTFSFASESTLNYCHSYSNILKYVCKTYFPASNREVYMK & \\
\hline 30.1 & 241 & HVNLDEINACFSRDGDKLTFSFASESTLNYSHSYSNILKYVCKTYFPASNREVYMKEFLVTRVNTWFCKFSRIDTFLLYK & \\
\hline 5.1 & 241 & HVNLDEINACFQRDGDRLTFSFASESTLNYTHSFSNILKYVCKTYFPASNREVYMKEFLVTRVNTWFCKFSRIDTFLLYK & 3 \\
\hline 07.1 & 321 & VNSEQFYSAMEDAWHYKKTLAMCNSERIL & \\
\hline 80.1 & 321 & GVAHKSVDSEQFYTAMEDAWHYKKTLAMCNSERILLEDSSSVNYWFPKMRDMVIVPLFDISLETSKRTRKEVLVSKDFVF & 4 \\
\hline 25.1 & 321 & GVAHKGVDSEQFYKAMEDAWHYKKTLAMCNSERILLEDSSSVNYWFPKMRDMVIVPLFDICLETSKRSRKEVLVSKDFVY & 40 \\
\hline & 91 & sv & \\
\hline 6880.1 & 401 & VLNHIRTYQAKALTYANVLSFVESIRSRVI INGVTARSEWDVDKSLLQSLSMTFFLHTKLAVLKDDLMISKFSFGPKTV & \\
\hline $\mathbf{X 3 2 6 2 5 . 1}$ & 401 & VLNHIRTYQAKALTYANVLSFVESIRSRVIINGVTARSEWDVDKSLLQSLSMTFFLHTKLSVLKDDLLISKFSLGPKPV & \\
\hline
\end{tabular}

\section{Fig. 9: COBALT output for Teal Cluster Set B}

\section{CONCLUSIONS}

V.

Hierachical clustering technique is able to identify similar groups based on Protein sequence. We able to identify similar groups eg.1: Tomato spotted wilt orthotospovirus, Chrysanthemum stem necrosis virus, Groundnut ringspot virus, Alstroemeria necrotic streak virus another example Rhizoctonia solani RNA virus HN008, Aspergillus fumigatus polymycovirus 1, Erysiphe necator associated deltaflexivirus 1, Alternaria alternata polymycovirus 1, Ingleside virus. All these possible due to Machine Learning techniques such as Hierarchical clustering method. These Methods or algorithms can be further improved to solve genomics and proteomics problems. 


\section{REFERENCES}

[1]. Venkataraman, S., Prasad, B., \& Selvarajan, R. (2018). RNA Dependent RNA Polymerases: Insights from Structure, Function and Evolution. Viruses, 10(2), 76. https://doi.org/10.3390/v10020076

[2]. [Internet]. https://www.displayr.com/what-is-hierarchical-clustering/

[3]. Nielsen, Frank (2016). "8. Hierarchical Clustering". Introduction to HPC with MPI for Data Science. Springer. pp. 195211. ISBN 978-3-319-21903-5.

[4]. Protein [Internet]. Bethesda (MD): National Library of Medicine (US), National Center for Biotechnology Information; 2021. Available from: https://www.ncbi.nlm.nih.gov/protein

[5]. Fabian Pedregosa, Gaël Varoquaux, Alexandre Gramfort, Vincent Michel, Bertrand Thirion, Olivier Grisel, Mathieu Blondel, Peter Prettenhofer, Ron Weiss, Vincent Dubourg, Jake Vanderplas, Alexandre Passos, David Cournapeau, Matthieu

Brucher, Matthieu Perrot, Édouard Duchesnay. Scikit-learn: Machine Learning in Python, Journal of Machine Learning Research, 12, 2825-2830 (2011

[6]. Pauli Virtanen, Ralf Gommers, Travis E. Oliphant, Matt Haberland, Tyler Reddy, David Cournapeau, Evgeni Burovski, Pearu Peterson, Warren Weckesser, Jonathan Bright, Stéfan J. van der Walt, Matthew Brett, Joshua Wilson, K. Jarrod Millman, Nikolay Mayorov, Andrew R. J. Nelson, Eric Jones, Robert Kern, Eric Larson, CJ Carey, İlhan Polat, Yu Feng, Eric W. Moore, Jake VanderPlas, Denis Laxalde, Josef Perktold, Robert Cimrman, Ian Henriksen, E.A. Quintero, Charles R Harris, Anne M. Archibald, Antônio H. Ribeiro, Fabian Pedregosa, Paul van Mulbregt, and SciPy 1.0 Contributors. (2020) SciPy 1.0: Fundamental Algorithms for Scientific Computing in Python. Nature Methods, 17(3), 261-272.

[7]. Chapman BA and Chang JT (2000). Biopython: Python tools for computational biology. ACM SIGBIO Newsletter, 20, $15-19$

[8]. [Internet]. https://biopython.org/docs/1.75/api/Bio.Entrez.html

[9]. [Internet]. https://www.kaggle.com/

[10]. Papadopoulos JS and Agarwala R (2007) COBALT: constraint-based alignment tool for multiple protein sequences, Bioinformatics 23:1073-79. PubMed.

[11]. [Internet]. https://www.ncbi.nlm.nih.gov/tools/cobalt/cobalt.cgi?

\section{ACKNOWLEDGMENT}

This paper and the research behind it would not have been possible without Python, Biopython, NCBI and Friend's support. 


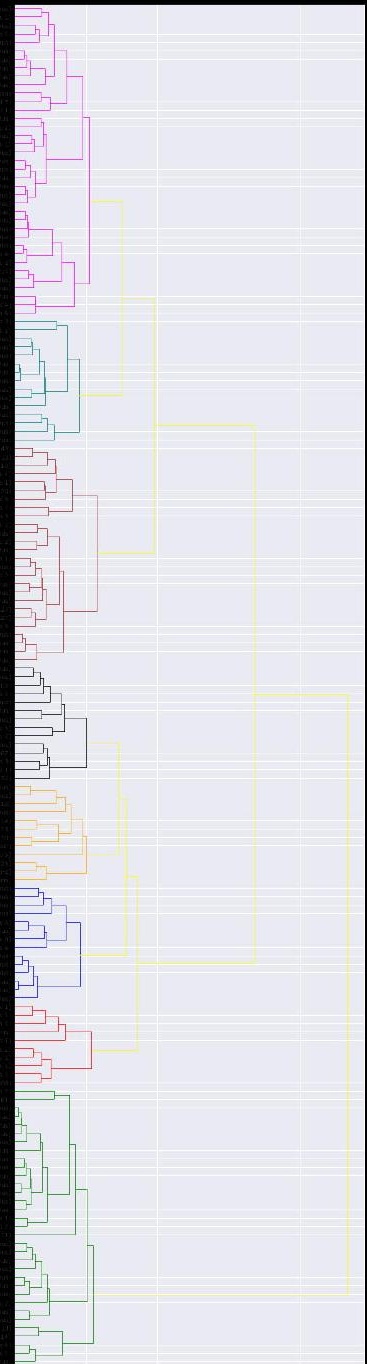

